\title{
Mesenchymal stem cells overexpressing integrin-linked kinase attenuate cardiac fibroblast proliferation and collagen synthesis through paracrine actions
}

\author{
QING MAO $^{1 *}$, CHENG-XI LIN $^{1 *}$, XIU-LIN LIANG ${ }^{2}$, JIAN-SHU GAO $^{1}$ and BIAO XU ${ }^{1}$ \\ ${ }^{1}$ Department of Cardiology, Drum Tower Hospital, Nanjing University Medical School, Nanjing 210008; \\ ${ }^{2}$ Department of Gerontology, The First People's Hospital of Changzhou, \\ The Third Affiliated Hospital of Suzhou University, Changzhou 213000, P.R. China
}

Received October 20, 2012; Accepted January 18, 2013

DOI: $10.3892 / \mathrm{mmr} .2013 .1348$

\begin{abstract}
Mesenchymal stem cells (MSCs) transfected by integrin-linked kinase (ILK) transplantation may improve the function and compliance of the post-infarct cardiac ventricle. We investigated the effect of ILK-modified MSC contiditioned medium (ILK-MSC-CM) on the proliferation of cardiac fibroblasts (CFBs) and collagen synthesis in vitro and in vivo. Myocardial infarction (MI)-induced animals received mesenchymal stem cell conditioned medium (MSC-CM), ILK-MSC-CM, or complete medium alone, subepicardially. A group of animals with $\mathrm{MI}$ and no other former intervention served as controls. ILK-MSC-CM inhibited CFB proliferation, reduced the gene expression of type I (Colla1) and type III collagen (Col3a1), tissue inhibitors of metalloproteinase-1 (TIMP-1) and -2 (TIMP-2), $\alpha$ smooth muscle actin ( $\alpha$-SMA), and connective tissue growth factor (CTGF). It also increased the gene expression of matrix metalloproteinase-2 (MMP-2) and -9 (MMP-9), as measured by qRT-PCR. Four weeks after the left anterior descending (LAD) coronary artery ligation, echocardiographic analysis demonstrated preserved cardiac geometry and contractility in the ILK-MSC-CM treated animals. Decreased infarct size and reduced fibrosis were observed in the ILK-MSC-CM group. Overexpression of ILK regulates paracrine actions of MSCs, and ILK-MSC-CM attenuates CFB proliferation and collagen synthesis through paracrine actions in vitro and in vivo.
\end{abstract}

Correspondence to: Professor Biao Xu, Department of Cardiology, Drum Tower Hospital, Nanjing University Medical School, Nanjing, Jiangsu 210008, P.R. China

E-mail: biaoxucn@126.com

*Contributed equally

Key words: integrin-linked kinase, mesenchymal stem cell, cardiac fibroblast, myocardial infarction, gene therapy

\section{Introduction}

Myocardial infarction (MI) leads to cardiomyocyte loss and scar formation. Damaged myocytes in infarcted tissues undergo progressive replacement by cardiac fibroblasts (CFBs) to form scar tissue that causes a remodeling of the left ventricle (LV). Consequently, cell-based therapeutic strategies for myocardial repair have recently attracted considerable interest, emerging as a promising cellular therapy for ischemic heart disease (IHD). Recently, a growing body of evidence has shown that mesenchymal stem cell (MSC) transplantation decreases fibrosis in the post-infarcted heart (1).

As a major cellular component of the heart, CFBs are directly involved in collagen synthesis of the infarct zone. One novel strategy to reduce scar formation after MI is pivotal to inhibit CFB proliferation and collagen synthesis. It has been shown that mesenchymal stem cell conditioned medium (MSC-CM) significantly attenuates CFB proliferation and collagen synthesis (2).

Bone marrow-derived MSCs are multipotent progenitor cells. They are easy to obtain and expand readily. They are also self-renewing and capable of repairing infarcted myocardium. Integrin-linked kinase (ILK) is a critical component of the cardiac mechanical stretch sensor. It not only plays an important role in maintaining myocardial contractility and function, it is also important in anti-apoptosis and angiogenesis. Recent studies have shown that in rat models, ILK-MSC delivery decreased the fibrotic heart area after MI (3). However, the potential mechanism of ILK-MSCs after MI is not yet fully elucidated.

Therefore, we hypothesized that ILK-MSCs attenuate CFB proliferation and collagen synthesis through paracrine effects. To test this hypothesis, we investigated the effects of ILK-MSC-CM on CFB proliferation and gene expression of cytokines. We implanted ILK-MSC-CM into infarcted hearts and analyzed the cardiac morphological and functional changes in the implanted and injured hearts.

\section{Materials and methods}

Ethics. This study was approved by the Ethics Committee of Nanjing Drum Tower Hospital, Nanjing, China (DTH ERBA $66.01 / 026 \mathrm{D} / 2010)$. 
Preparation and adenoviral transduction of MSCs. MSCs were isolated from the bone marrow of Sprague-Dawley rats and were expanded according to previously reported protocols (4). The cells were suspended in Dulbecco's modified Eagle medium-low glucose (DMEM-LG; Gibco, Carlsbad, CA, USA) containing $10 \%$ fetal bovine serum (FBS; Gibco), $100 \mathrm{U} / \mathrm{ml}$ penicillin and $100 \mu \mathrm{g} / \mathrm{ml}$ streptomycin. Fourth-passage cells were transduced with a recombinant adenoviral vector harboring human wild-type ILKcDNA and humanized recombinant green fluorescent protein (hrGFP). Transduction efficiency was assessed by fluorescence-activated cell sorting (FACS) analysis (Becton-Dickinson, Franklin Lakes, NJ, USA), western blotting, real-time PCR and immunofluorescence.

Preparation of CFBs. CFBs were prepared and cultured as previously described with minimum modification $(5,6)$.

Treatment of CFBs with conditioned medium. Conditioned medium was collected from a 72-h culture of fourth-passage MSCs and ILK-modified MSCs (ILK-MSCs) and normalized by cultured cell numbers. After removing the cell debris, the supernatant was transferred into dedicated ultrafiltration tubes (Amicon Ultra-PL 5; Millipore, Billerica, MA, USA), concentrated and desalted according to the manufacturer's protocol.

CFBs were incubated in MSC-CM or ILK-MSC-CM for $72 \mathrm{~h}$. The control group was incubated in DMEM-high glucose (DMEM-HG) containing 5\% FBS for the same period of time.

$R T-P C R$. Total RNA was extracted from cultured cells using RNAprep pure cell kit (Tiangen, Beijing, China). RNA quality was assessed by the ratio of absorbance at 260 and $280 \mathrm{~nm}$. One microgram of total RNA was used for first strand cDNA synthesis by Reverse Transcriptase from Invitrogen. Quantitative real-time PCR was carried out using the ABI Prism 7000 sequence detection system (Applied Biosystems) with SYBR green (Applied Biosystems). All samples were plated in 48-well plates with each well containing $1 \mu \mathrm{l}$ cDNA for a total reaction volume of $10 \mu \mathrm{l}$. Reactions were carried out at $95^{\circ} \mathrm{C}$ for $10 \mathrm{~min}$, followed by 40 cycles of $95^{\circ} \mathrm{C}$ for $5 \mathrm{sec}$ and $60^{\circ} \mathrm{C}$ for $34 \mathrm{sec}$. Glyceraldehyde 3 -phosphate dehydrogenase (GAPDH) mRNA amplified from the same samples served as an internal control. Primers were designed by using Primer 3 software. Primer sequences were: ILK, forward: 5'-GATTGTGCCTATCCTTGAGAAGAT-3' and reverse: 5'-TAAACTTTATTGTGACAGGCGG-3'; CTGF, forward: 5'-GGCTGGAGAAGCAGAGTCGT-3' and reverse: 5'-GATGCACTTTTTGCCCTTCTTAA-3'; $\alpha$-SMA, forward: 5'-TTCGTTACTACTGCTGAGCGTGAGA-3' and reverse: 5'-AAGGATGGCTGGAACAGGGTC-3'; Col1a1, forward: 5'-ATCAGCCCAAACCCCAAGGAGA-3' and reverse: 5'-CGCAGGAAGGTCAGCTGGATAG-3'; Col3a1, forward: 5'-TGATGGGATCCAATGAGGGAGA-3' and reverse: 5'-GAGTCTCATGGCCTTGCGTGTTT-3'; MMP-2, forward: 5'-GATCTGCAAGCAAGACATTGTCTT-3' and reverse: 5'-GCCAAATAAACCGATCCTTGAA-3'; MMP-9, forward: 5'-CGCAAGCCTCTAGAGACCAC-3' and reverse: 5'-TGGGGGATCCGTGTTTATTA-3'; TIMP-1, forward: 5'-CTGCAACTCGGACCTGGTTATAAGG-3' and reverse: 5'-AACGGCCCGCGATGAGAAACTCC-3'; TIMP-2, forward: 5'-ATTCCGGGAATGACATCTATGGCAAC-3' and reverse: 5'-CTGGTACCTGTGGTTTAGGCTCTTC-3'; GAPDH, forward: 5'-GGGGTGAGGCCGGTGCTGAGTA-3' and reverse: 5'-CATTGGGGGTAGGAACACGGAAGG-3'. Each experiment was repeated three times.

Western blotting. To identify ILK protein expression, western blotting was performed with rabbit antibodies against ILK. MSCs infected with Ad-ILK-hrGFP were cultured with complete medium for $72 \mathrm{~h}$. After a brief wash with PBS, cells were lysed in $0.1 \%$ Triton-X 100, $10 \mathrm{mM}$ TRIS pH 8, $1 \mathrm{mM}$ EDTA, and supplemented with a protease and phosphatase inhibitor cocktail. Proteins were separated by $10 \%$ TRIS-HCL gel electrophoresis (Bio-Rad), resolved onto $0.2 \mu \mathrm{m}$ nitrocellulose membranes, and blocked in nonfat milk in TRIS-buffered saline supplemented with $0.1 \%$ Tween-20. Immunoreactivity against ILK or GAPDH was visualized with appropriate horseradish-peroxidase-conjugated antibodies and ECL plus Western Blotting Detection Reagents. Densitometric analysis was performed with Abode Photoshop. The ILK signals were normalized to signals of GAPDH. Experiments were performed in triplicate and repeated three times.

5-ethynyl-2'-deoxyuridine (EdU) staining. EdU staining was performed using Click-iT ${ }^{\circledR}$ EdU Imaging kit (cat no. Cc103338; Invitrogen, Carlsbad, CA, USA) according to the manufacturer's protocol.

Acute myocardial infarction. Ligation of the LAD artery was performed as previously described (7). Briefly, animals were anesthetized by intraperitoneal injection with a combination of xylazine $(10 \mathrm{mg} / \mathrm{kg})$ and ketamine $(80 \mathrm{mg} / \mathrm{kg})$, endotracheally intubated, and mechanically ventilated. After a left thoracotomy through the fifth intercostal space, the heart was exposed, the pericardial sac was cut, and the heart exteriorized through the space. The LAD was ligated with a 6-0 silk suture approximately midway between the left atrium and the apex of the heart. Successful MI was identified by blanching of the left ventricular muscle and the presence of ST elevation on electrocardiograms.

Prior to surgery, animals were randomized into four groups. Accordingly, a total volume of $600 \mu \mathrm{l}$ of MSC-CM, ILK-MSC-CM or complete medium was injected in five different sites at the infarct border zone (BZ) 30 min after LAD ligation. Control animals were operated at the same way, with the exception that no injection was performed. The chest was closed, and animals were weaned from the ventilator and allowed to recover.

Echocardiography. Echocardiographic investigation was performed three days and 4 weeks after MI.

Histological analysis. Animals were sacrificed, and cardiovascular tissues were collected at the end of the experiment. Paraffin-embedded sections (from basal, mid-region and apical blocks: 3 slices/block) were stained with hematoxylin and eosin (H\&E) for morphological examination or Masson's trichrome staining was performed for the assessment of interstitial fibrosis. 

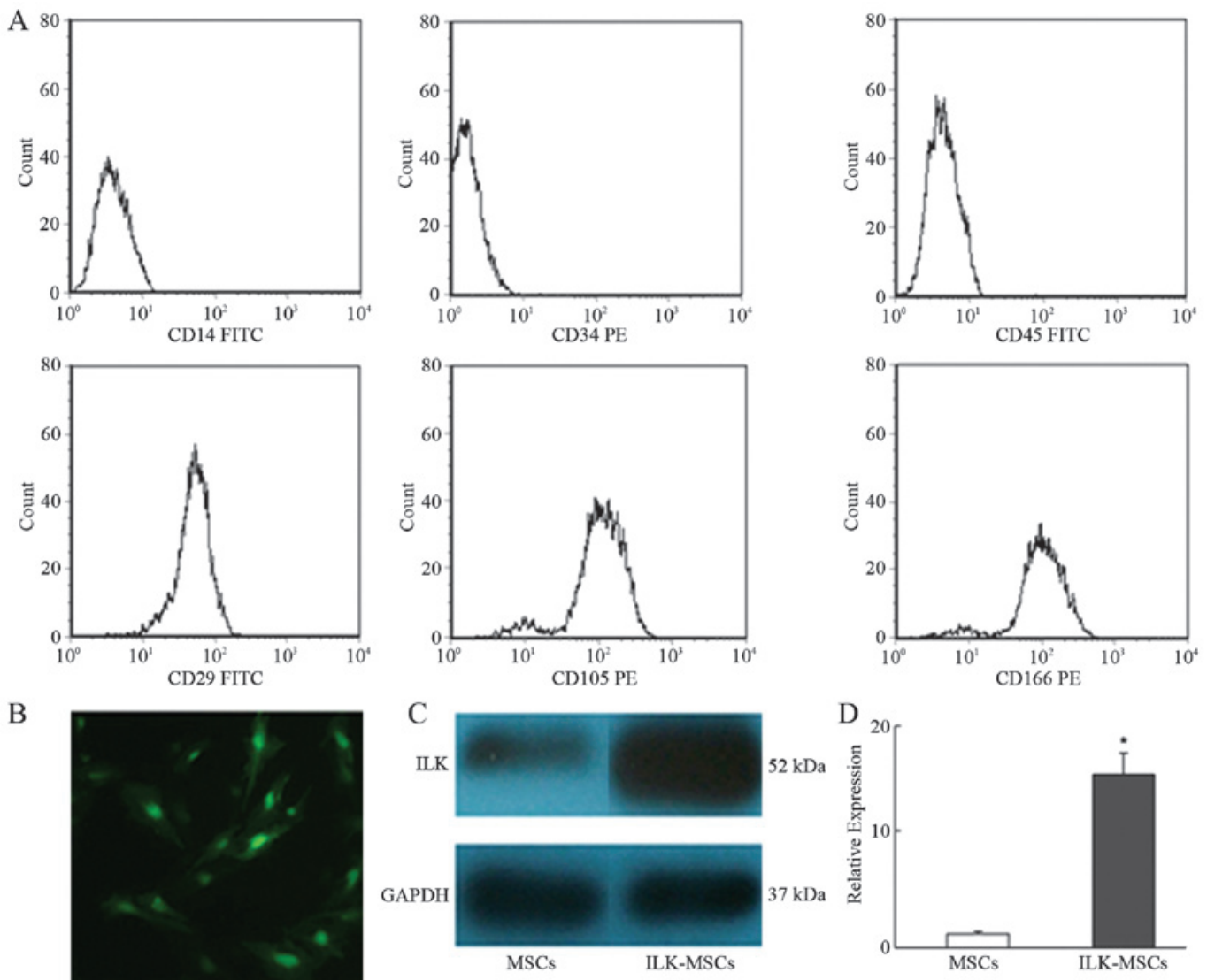

Figure 1. Phenotypic analysis and transfection of mesenchymal stem cells (MSCs) with Ad-ILK-hrGFP in vitro. (A) MSCs were uniformly labeled with antibodies against the indicated antigens analyzed with flow cytometry. Most of the cells were positive for CD29, CD105 and CD166, but negative for CD14, CD34 and CD45. (B) Representative photomicrograph of MSCs transfected with Ad-ILK-hrGFP. ILK-MSCs were consistent with the shapes of the MSCs. The GFP-labeled cells emitted green fluorescence after transduction for $48 \mathrm{~h}$. Original magnification, x100. (C) Representative western blotting showing that integrin-linked kinase (ILK) was overexpressed in ILK-MSCs. (D) Real-time PCR showing that ILK was overexpressed in ILK-MSCs. "P<0.05 vs. MSCs.

Statistical analysis. Statistical analysis was performed with SPSS for Windows (version 16.0). All the data were expressed as the means \pm standard error (SE). Siginificant differences among experimental conditions were determined by two-tailed Student's t-tests for two-group comparisons or ANOVA followed by Newman-Keuls test for multiple-group comparisons. $\mathrm{P}<0.05$ was considered to indicate a statistically significant difference.

\section{Results}

Transduction of MSCs with adenoviral vector expressing ILK. FACS analysis demonstrated that MSCs in culture expressed low levels of CD14, CD34 and CD45, while high levels of CD29, CD105 and CD166, in agreement with previously published data regarding MSC cell surface markers (Fig. 1A).

The viral transduction efficacy was $85-95 \%$ in MSCs infected with Ad-ILK-hrGFP at 200 MOI and cultured for $72 \mathrm{~h}$. The expression of hrGFP in ILK-MSCs was confirmed by photomicrograph, qRT-PCR and western blotting (Fig. 1B-D).

Decrease of the quantity of EdU-positive CFBs. Our results showed that the percentage of CFBs positively stained for EdU was significantly decreased in both MSC-CM and ILK-MSC-CM groups. The relative number of EdU-positive cells was $41 \pm 2,29 \pm 4$ and $22 \pm 3 \%$ for the control, MSC-CM and ILK-MSC-CM groups, respectively. The relative number of EdU-positive cells showed a $\sim 30$ and $46 \%$ decrease in the MSC-CM and ILK-MSC-CM group compared to the control group. In addition, the ILK-MSC-CM group showed a $\sim 25 \%$ decrease in the percentage of EdU-positive cells compared to the MSC-CM group (Fig. 2A and B).

Effect of conditioned medium on cytokine gene expression. The results demonstrated that gene expression of Colla1, Col3a1, TIMP-1, TIMP-2, $\alpha$-SMA and CTGF was significantly downregulated when CFBs were cultured in conditioned medium compared to standard medium. However, MMP-2 and MMP-9 gene expression in CFBs incubated with conditioned medium was significantly upregulated. The TIMP-1, TIMP-2, $\alpha$-SMA and CTGF gene expression levels of CFBs were significantly lower in the ILK-MSC-CM-treated compared to the MSC-CM-treated cells. ILK-MSC-CM reduced Col3a1 to a level lower compared to MSC-CM. ILK-MSC-CM significantly upregulated MMP-2 expression, while it downregulated MMP-9 expression compared to MSC-CM (Fig. 2C-F and H-J).

Cardiac function as detected by echocardiography. After the ligation of the LAD artery, the MI model detected by echocardiography was successfully established. Three days and 4 weeks after MI, LV ejection fraction (EF), percent LV fractional shortening (\%FS), and interventricular septal thick- 

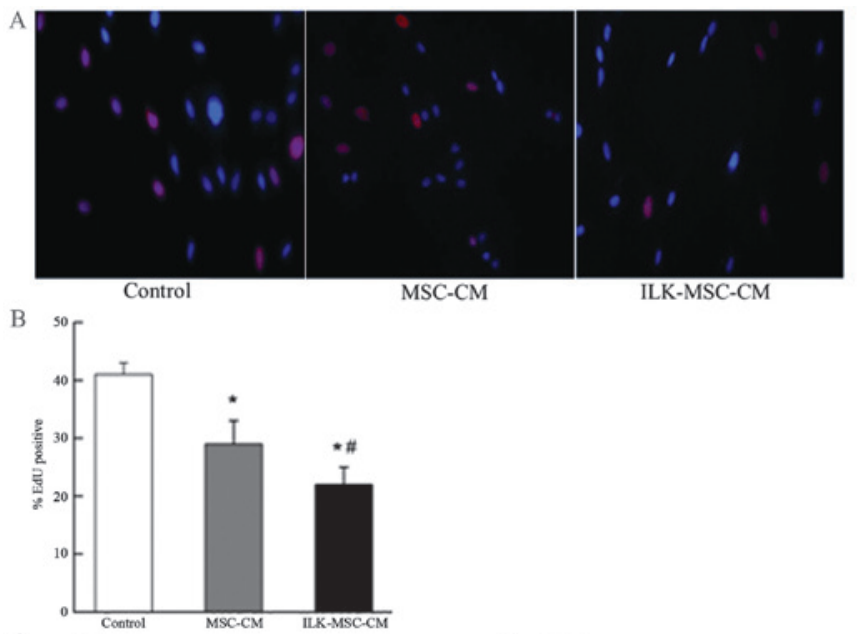

$\mathrm{C}$

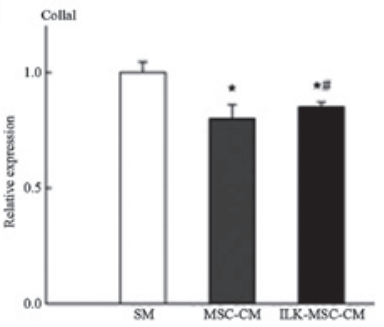

E

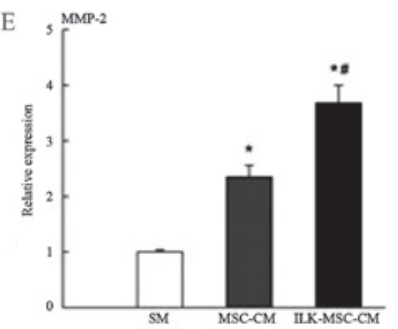

G
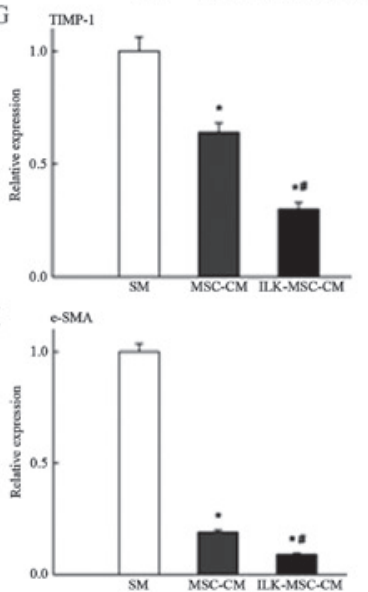

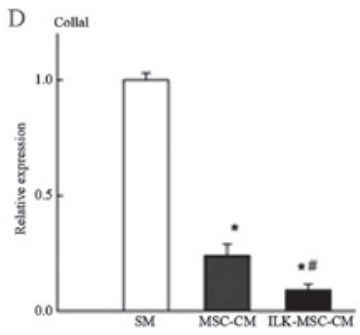

F

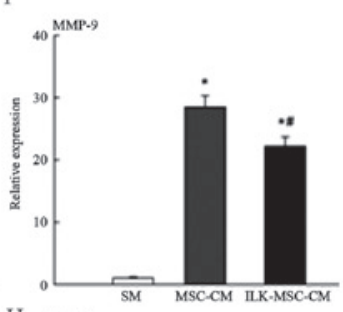

$\mathrm{H}$ TIMP-2
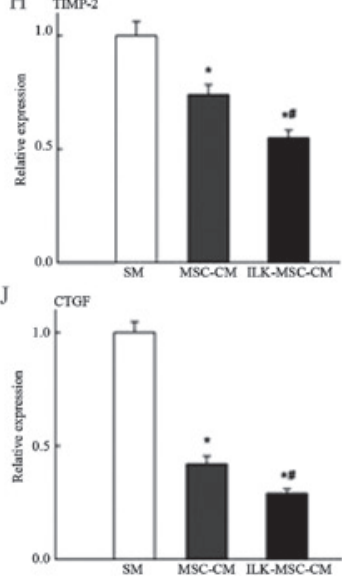

Figure 2. Effects of ILK-MSC-CM on CFB proliferation and gene expression of cytokines. (A) Representative imaging showing that the ILK-MSC-CM group contained fewer EdU-positive cells than the MSC-CM and control groups. EdU (red), Hoechst 33342 (blue). Original magnification, x200. (B) Quantification of EdU-positive CFBs from 10 fields of view. Data are represented as the number of EdU-positive cells relative to the total number of cells. Real-time PCR for (C) Colla1, (D) Col3a1, (E) MMP-2, (F) MMP-9, (G) TIMP-1, (H) TIMP-2, (I) $\alpha$-SMA, (J) CTGF gene expression of CFBs after $72 \mathrm{~h}$ of culture in the indicated medium. Values are the means $\pm \mathrm{SE}$. ${ }^{*} \mathrm{P}<0.05$ vs. $\mathrm{SM} ;{ }^{\#} \mathrm{P}<0.05$ vs. MSC-CM. Each group $\mathrm{n}=3$. ILK, integrin-linked kinase; MSC-CM, mesenchymal stem cell-conditioned medium; SM, standard medium; CFB, cardiac fibroblasts.

ness in diastole (IVSd) demonstrated significant improvement in the ILK-MSC-CM group compared to the control, complete
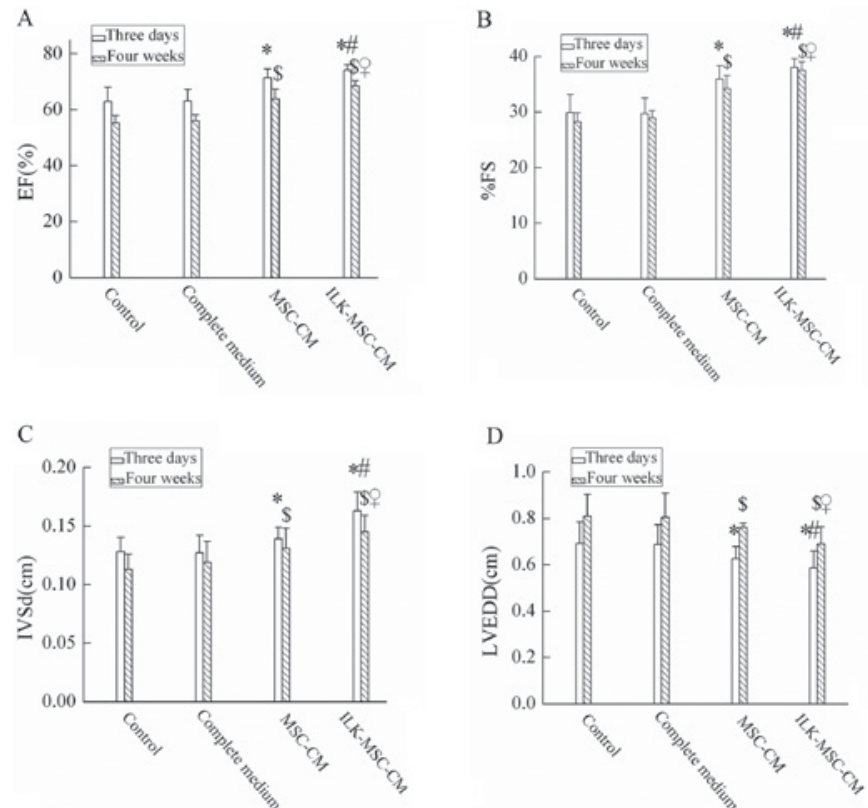

$\mathrm{E}$

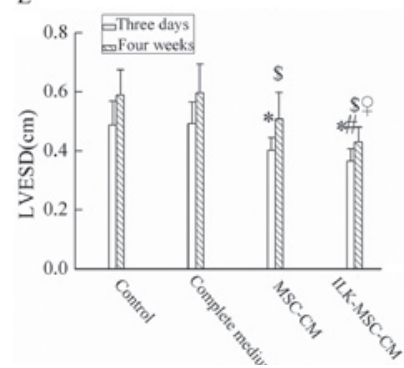

Figure 3. Echocardiographic evaluation of LV ejection fraction (EF), percent LV fractional shortening (\%FS), and interventricular septal thickness in diastole (IVSd), LV end-diastolic dimension (LVEDD) and LV end-systolic dimension (LVESD). Quantification by echocardiographic analysis of (A) EF (B) \%FS and (C) IVSd. EF, \%FS and IVSd showed significant improvement in the ILK-MSC-CM group three days and 4 weeks after myocardial infarction (MI). Quantification by echocardiographic analysis of the (D) LVEDD and (E) LVESD. There was a marked reduction of LVESD and LVEDD in the ILK-MSC-CM group. Values are the means $\pm \mathrm{SE}$. ${ }^{*} \mathrm{P}<0.05$ vs. control three days after MI. ${ }^{\#} \mathrm{P}<0.05$ vs. MSC-CM group three days after $\mathrm{MI} .{ }^{\$} \mathrm{P}<0.05$ vs. control four weeks after MI. ${ }^{\circ} \mathrm{P}<0.05$ vs. MSC-CM group four weeks after MI. Each group n=20. ILK, integrin-linked kinase; MSC-CM, mesenchymal stem cell-conditioned medium.

medium and MSC-CM groups (Fig. 3A-C). In parallel with this, LV end-systolic dimension (LVESD) and LV end-diastolic dimension (LVEDD) were lower in the ILK-MSC-CM group (Fig. 3D and E). No significant difference in LV posterior wall thickness (LVPW) was detected.

Histological analysis. The effect of ILK-MSC-CM on myocardial injury 4 weeks after infarction was evaluated by $\mathrm{H} \& \mathrm{E}$ staining. The mean infarct size in the control animals was $35 \pm 3 \%$ of the LV. Injection of complete medium had a similar injury effect, limiting the size of the infarct to $36 \pm 4 \%$ of the LV. However, injection of MSC-CM had a modest protective effect, limiting the size of the infarct to $30 \pm 4 \%$ of the LV. By contrast, injection of ILK-MSC-CM significantly limited the infarct size to $22 \pm 3 \%$ of the LV. Compared to the other three groups, this translated into a relative reduction in the infarct size of the ILK-MSC-CM group (Fig. 4A-D). 

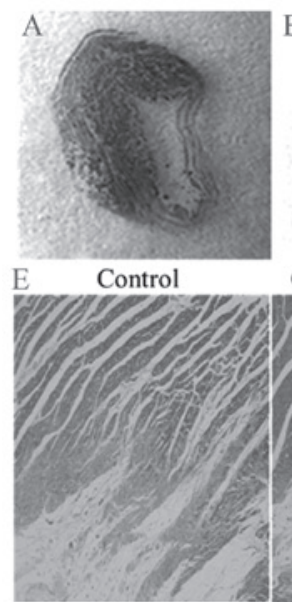

Control

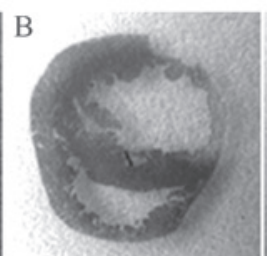

Complete medium

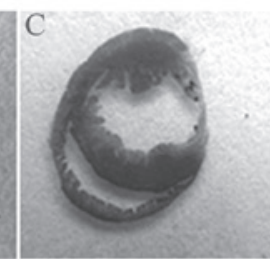

MSC-CM

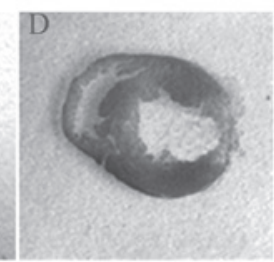

ILK-MSC-CM

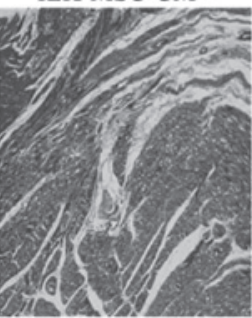

ILK-MSC-CM

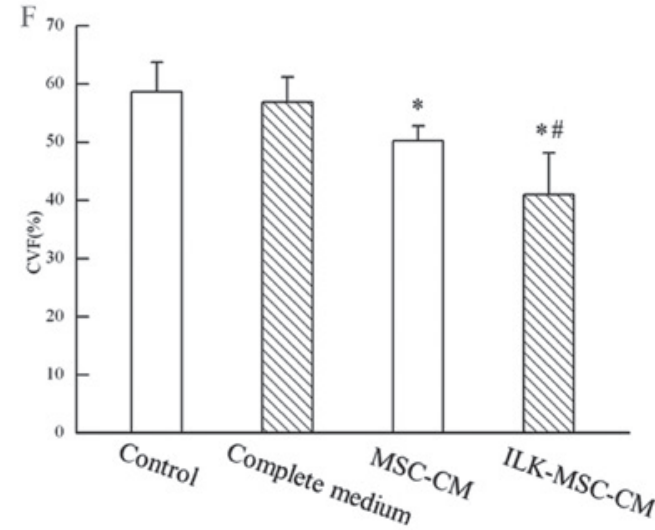

Figure 4. Effect of the ILK-MSC-CM on infarct size and fibrosis in the infarcted hearts. (A) The LAD ligation in the control group resulted in an infarct size equivalent in size to a third of the entire left ventricular area as documented by H\&E staining. (B) After injection of complete medium, infarct size was similar to that of the control group. (C) After injection of MSC-CM, infarct size was reduced but not significantly. (D) Treatment with ILK-MSC-CM remarkably limited the infarct size. (E) Masson's Trichrome staining in control, complete medium, MSC-CM and ILK-MSC-CM groups (magnification, x400). (F) Quantitative analysis of Masson's Trichrome stained myocardial area showed significantly decreased fibrosis in the ILK-MSC-CM group compared with control, the complete medium and MSC-CM groups. ILK, integrin-linked kinase; MSC-CM, mesenchymal stem cell-conditioned medium. ${ }^{*}<0.05$ vs. control. ${ }^{\#} \mathrm{P}<0.05$ vs. MSC-CM group.

Reduction of interstitial fibrosis in infarct boder zone. Masson's trichrome staining for interstitial fibrosis in the infarct BZ in the four experimental groups is shown in Fig. 4E. Collagen volume fractions (CVF) were significantly lower in the ILK-MSC-CM group compared to the other three groups. Consequently, ILK-MSC-CM treatment significantly inhibits collagen synthesis and suppresses collagen deposition, resulting in a reduction in ventricular remodeling (Fig. 4F).

\section{Discussion}

In this present study, we studied the paracrine effects of ILK-MSCs on CFBs in vitro and in vivo. The following were demonstrated: i) MSC-CM and ILK-MSC-CM significantly inhibited CFB proliferation, while ILK-MSC-CM further suppressed CFB proliferation compared to MSC-CM. ii) MSC-CM and ILK-MSC-CM distinctly inhibited type I and III collagen gene ex pression in CFBs.ILK-MSC-CM further restrained type III collagen gene expression. iii) MSC-CM and ILK-MSC-CM significantly increased MMP-2 and MMP-9 gene expression in CFBs. iv) MSC-CM and ILK-MSC-CM significantly decreased TIMP-1 and TIMP-2 gene expression in CFBs. v) MSC-CM and ILK-MSC-CM decreased $\alpha$-SMA and CTGF gene expression in CFBs. ILK-MSC-CM further suppressed $\alpha$-SMA and CTGF gene expression compared to MSC-CM. (6) More significant improvement occurred regarding the infarct area, cardiac geometry (LVEDD, LVESD and IVSd), contractility (EF, \%FS) and pathological changes of the infarcted heart with ILK-MSC-CM transplantation.

In the mammalian heart, normal cardiac function is regulated by concordant and dynamic interactions of two major cell types, cardiomyocytes and CFBs, which account for $\sim 90 \%$ of all the cells in the myocardium. The extracellular matrix (ECM) regulates the structure of the heart and interactions between cellular and non-cellular components. In the normal heart, CFBs are mainly recognized as regulators of ECM metabolism, thereby maintaining myocardial structure. These cells control ECM homeostasis and produce MMPs and TIMPs to maintain a balance between the synthesis and degradation of ECM components. CFBs also play a pivotal role in the remodeling that occurs in the heart in response to pathological changes, such as MI, heart failure and hypertension.

CFBs have the ability to synthesize and secrete collagen type I and III and form a three-dimensional network around bundles or laminae of myocytes (8). Inhibition of the collagen network formation results in abnormally shaped hearts or rupture of the ventricular wall. Under circumstances that accompany myocardial remodeling, normally quiescent CFBs undergo phenotypic modulation to myofibroblasts. In the remodeled heart, myofibroblasts become highly proliferative and invasive, actively remodeling the cardiac interstitium by increasing MMP secretion and collagen turnover (9-11). CFBs, by themselves, secrete increased amounts of growth factors and cytokines, which are able to activate MMPs and regulate TIMPs, thereby contributing to remodeling. In long-term heart remodeling, cumulative changes lead to a net accumulation of collagen, cardiac fibrosis and loss of cardiac function. Therefore, previous studies have shown that myocardial scar tissue formation eventually leads to congestive heart failure and malignant arrhythmias (2). In this respect, reduction of fibrosis improves the ventricular compliance to a certain extent and delays the process of heart failure.

Bone marrow-derived MSCs are self-renewing, multipotent, and can differentiate into several distinct cell types, including new cardiomyocytes. MSC transplantation has been shown to improve cardiac function and decrease fibrosis in the heart and other organs such as the lung, liver and kidney. Previous studies have demonstrated that paracrine effects may contribute to the improvement of cardiac function and scar loss following transplantation of MSCs (12-14). For instance, 
MSC-CM has been shown to significantly attenuate the proliferation of CFBs compared to CFB-conditioned medium (2). However, the underlying mechanisms of MSC therapy remain controversial.

ILK is a 59-kDa ankyrin-repeat containing serine/threonine protein kinase that interacts with the cytoplasmic domain of $\alpha 1$ and $\alpha 3$ integrins and regulates integrin-dependent functions (15-17). ILK functions as a scaffold in forming multiprotein complexes connecting integrins to the actin cytoskeleton and signaling pathways. ILK activity is stimulated by adhesion to the ECM and by growth factors in a PI3K-dependent manner. ILK regulates multiple signaling pathways and has been implicated in the regulation of numerous cellular processes including proliferation, migration, cell spreading, invasion, differentiation, transformation and survival in various cell types. Recent evidence have shown that ILK-MSC transplantation further improves cardiac function and decreases fibrosis in the infarcted myocardium when compared with myocardium that has been transplanted with MSCs alone (18). However, there is still a lack of related experiments on whether ILK-MSCs play an important role through paracrine mechanisms to improve ventricular remodeling.

Many of the functional effects of CFBs are mediated through differentiation of CFBs to myofibroblasts, cells that express contractile proteins, including $\alpha$-SMA, a myofibroblast marker. Differentiating fibroblasts express $\alpha$-SMA, indicating acquisition of a secretory, myofibroblast phenotype, a transition that correlates with increased secretion of profibrotic molecules such as collagen and fibronectin $(19,20)$. The present study demonstrated that ILK-MSC-CM downregulates $\alpha$-SMA expression and suppresses the differentiation of CFBs to myofibroblasts.

CTGF is a $38-\mathrm{kDa}$ protein that contains 38 conserved cysteine residues and a heparine binding domain (21). Recently, it has been reported that CTGF promotes proliferation and ECM production in connective tissue $(22,23)$. This study demonstrated that ILK-MSC-CM inhibits CTGF expression, which is beneficial to ECM degradation.

MMP-2 and MMP-9 are two members of the MMP family, a group of zinc-dependent endopeptidases known to hydrolyze many components of the ECM (24). MMP-2 and MMP-9 play a fundamental role in ECM remodeling due to their ability to initiate and continue degradation of fibrillar collagen.

Belonging to a group of four endogenous proteins, TIMPs firmly regulate MMP activity $(25,26)$. TIMPs bind to the active site of MMPs at a stoichiometric 1:1 molar ratio with differential affinities, thereby blocking access to their extracellular substrates. Several studies have shown that TIMP-2 has a maximum affinity for MMP-2, whereas TIMP-1 forms a specific complex with pro-MMP-9 (27-29). In normal cardiac tissue, MMPs and TIMPs are co-expressed and tightly regulated to maintain integrity of the cardiac interstitium (30). Consequently, the MMP/TIMP system is a crucial contributor to ECM turnover and heart failure progression.

In the present study, it was found that the MMP-9/TIMP-1 ratio was not differentiated in MSC and ILK-MSC groups, whereas the MMP-2/TIMP-2 ratio significantly increased in ILK-MSC compared to the MSC group. Elevation of the MMP-2/TIMP-2 ratio was conducive to the degradation of collagen. Furthermore, it was favorable to angiogenesis and heart function improvement accompanied by escalation of MMP-2 and MMP-9.

In conclusion, the data presented here demonstrate that ILK-MSCs exert paracrine anti-fibrotic effects at least in part through inhibition of CFB proliferation and downregulation of collagen synthesis. The overexpression of ILK is able to regulate the partial paracrine effects of MSCs. These features of ILK-MSCs could be beneficial for the treatment of heart failure in which fibrotic changes are involved.

\section{Acknowledgements}

The authors would like to thank Professor Zheng Xing from the University of Minnesota for expert technical assistance, and Congzhu Shi from the University of Pennsylvania for helpful comments and suggestions.

\section{References}

1. Jaquet K, Krause KT, Denschel J, et al: Reduction of myocardial scar size after implantation of mesenchymal stem cells in rats: what is the mechanism? Stem Cells Dev 14: 299-309, 2005.

2. Ohnishi S, Sumiyoshi H, Kitamura S and Nagaya N: Mesenchymal stem cells attenuate cardiac fibroblast proliferation and collagen synthesis through paracrine actions. FEBS Lett 581: 3961-3966, 2007.

3. Song SW, Chang W, Song BW, et al: Integrin-linked kinase is required in hypoxic mesenchymal stem cells for strengthening cell adhesion to ischemic myocardium. Stem Cells 27: 1358-1365, 2009.

4. Iwase T, Nagaya N, Fujii T, et al: Comparison of angiogenic potency between mesenchymal stem cells and mononuclear cells in a rat model of hindlimb ischemia. Cardiovasc Res 66: 543-551, 2005.

5. Chen K, Chen J, Li D, Zhang X and Mehta JL: Angiotensin II regulation of collagen type I expression in cardiac fibroblasts: modulation by PPAR-gamma ligand pioglitazone. Hypertension 44: 655-661, 2004

6. Frangogiannis NG, Dewald O, Xia Y, et al: Critical role of monocyte chemoattractant protein-1/cc chemokine ligand 2 in the pathogenesis of ischemic cardiomyopathy. Circulation 115: 584-592, 2007.

7. Li W, Ma N, Ong LL, et al: Bcl-2 engineered MSCs inhibited apoptosis and improved heart function. Stem Cells 25: 2118-2127, 2007.

8. Goldsmith EC, Hoffman A, Morales MO, et al: Organization of fibroblasts in the heart. Dev Dyn 230: 787-794, 2004.

9. Weber KT: Fibrosis in hypertensive heart disease: focus on cardiac fibroblasts. J Hypertens 22: 47-50, 2004.

10. Brown RD, Ambler SK, Mitchell MD and Long CS: The cardiac fibroblast: therapeutic target in myocardial remodeling and failure. Annu Rev Pharmacol Toxicol 45: 657-687, 2005.

11. Camelliti P, Borg TK and Kohl P: Structural and functional characterisation of cardiac fibroblasts. Cardiovasc Res 65: 40-51, 2005.

12. Dai W, Hale SL and Kloner RA: Role of a paracrine action of mesenchymal stem cells in the improvement of left ventricular function after coronary artery occlusion in rats. Regen Med 2: 63-68, 2007.

13. Li L, Zhang S, Zhang Y, Yu B, Xu Y and Guan Z: Paracrine action mediate the antifibrotic effect of transplanted mesenchymal stem cells in a rat model of global heart failure. Mol Biol Rep 36: 725-731, 2009.

14. Xu RX, Chen X, Chen JH, Han Y and Han BM: Mesenchymal stem cells promote cardiomyocyte hypertrophy in vitro through hypoxia-induced paracrine mechanisms. Clin Exp Pharmacol Physiol 36: 176-180, 2009.

15. Attwell S, Mills J, Troussard A, Wu C and Dedhar S: Integration of cell attachment, cytoskeletal localization, and signaling by integrin-linked kinase (ILK), CH-ILKBP, and the tumor suppressor PTEN. Mol Biol Cell 14: 4813-4825, 2003.

16. Tan C, Mui A and Dedhar S: Integrin-linked kinase regulates inducible nitric oxide synthase and cyclooxygenase-2 expression in an NF-kappa B-dependent manner. J Biol Chem 277: 3109-3116, 2002. 
17. Troussard AA, McDonald PC, Wederell ED, et al: Preferential dependence of breast cancer cells versus normal cells on integrin-linked kinase for protein kinase B/Akt activation and cell survival. Cancer Res 66: 393-403, 2006.

18. Ding L, Dong L, Chen X, et al: Increased expression of integrin-linked kinase attenuates left ventricular remodeling and improves cardiac function after myocardial infarction. Circulation 120: 764-773, 2009.

19. Gabbiani G: Evolution and clinical implications of the myofibroblast concept. Cardiovasc Res 38: 545-548, 1998.

20. Petrov VV, Fagard RH and Lijnen PJ: Stimulation of collagen production by transforming growth factor-beta 1 during differentiation of cardiac fibroblasts to myofibroblasts. Hypertension 39: 258-263, 2002

21. Chen MM, Lam A, Abraham JA, Schreiner GF and Joly AH: CTGF expression is induced by TGF-beta in cardiac fibroblasts and cardiac myocytes: A potential role in heart fibrosis. J Mol Cell Cardiol 32: 1805-1819, 2000.

22. Frazier K, Williams S, Kothapalli D, Klapper H and Grotendorst GR: Stimulation of fibroblast cell growth, matrix production, and granulation tissue formation by connective tissue growth factor. J Invest Dermatol 107: 404-411, 1996.

23. Igarashi A, Okochi H, Bradham DM and Grotendorst GR Regulation of connective tissue growth factor gene expression in human skin fibroblasts and during wound repair. Mol Biol Cell 4: 637-645, 1993.

24. Olson MW, Gervasi DC, Mobashery S and Fridman R: Kinetic analysis of the binding of human matrix metalloproteinase- 2 and -9 to tissue inhibitor of metalloproteinase (TIMP)-1 and TIMP-2 . J Biol Chem 272: 29975-29983, 1997.
25. Vanhoutte D, Schellings M, Pinto Y and Heymans S: Relevance of matrix metalloproteinases and their inhibitors after myocardial infarction: A temporal and spatial window. Cardiovasc Res 69: 604-613, 2006.

26. Brew K, Dinakarpandian D and Nagase H: Tissue inhibitors of metalloproteinases: evolution, structure and function. Biochim Biophys Acta 1477: 267-283, 2000.

27. Menon B, Singh M and Singh K: Matrix metalloproteinases mediate beta-adrenergic receptor-stimulated apoptosis in adult rat ventricular myocytes. Am J Physiol Cell Physiol 289: C168-C176, 2005.

28. Goldberg GI, Marmer BL, Grant GA, Eisen AZ, Wilhelm S and He CS: Human 72-kilodalton type IV collagenase forms a complex with a tissue inhibitor of metalloproteases designated TIMP-2. Proc Natl Acad Sci USA 86: 8207-8211, 1989.

29. Goldberg GI, Strongin A, Collier IE, Genrich LT and Marmer BL: Interaction of 92-kDa type IV collagenase with the tissue inhibitor of metalloproteinases prevents dimerization, complex formation with interstitial collagenase, and activation of the proenzyme with stromelysin. J Biol Chem 267: 4583-4591, 1992.

30. Brown RD, Jones GM, Laird RE, Hudson P and Long CS: Cytokines regulate matrix metalloproteinases and migration in cardiac fibroblasts. Biochem Biophys Res Commun 362: 200-205, 2007 